\title{
Distribution of Nanoparticles in a Turbulent Taylor-Couette Flow Considering Particle Coagulation and Breakage
}

\author{
Ruifang Shi, Jianzhong Lin *(D) and Hailin Yang $\mathbb{D}$ \\ State Key Laboratory of Fluid Power Transmission and Control, Zhejiang University, Hangzhou 310027, China; \\ shiruifang@zju.edu.cn (R.S.); yanghailin@zju.edu.cn (H.Y.) \\ * Correspondence: mecjzlin@public.zju.edu.cn
}

check for updates

Citation: Shi, R.; Lin, J.; Yang, $\mathrm{H}$ Distribution of Nanoparticles in a Turbulent Taylor-Couette Flow Considering Particle Coagulation and Breakage. Processes 2021, 9, 1789. https://doi.org/10.3390/pr9101789

Academic Editors: Haiping Zhu and Chandana Jayasundara

Received: 19 September 2021

Accepted: 6 October 2021

Published: 8 October 2021

Publisher's Note: MDPI stays neutral with regard to jurisdictional claims in published maps and institutional affiliations.

Copyright: (c) 2021 by the authors. Licensee MDPI, Basel, Switzerland. This article is an open access article distributed under the terms and conditions of the Creative Commons Attribution (CC BY) license (https:// creativecommons.org/licenses/by/ $4.0 /)$.

\begin{abstract}
In this paper, the dynamic evolution of nanoparticles in a turbulent Taylor-Couette flow was studied by means of a numerical simulation. The initial particle size was $200 \mathrm{~nm}$, and the volume concentration was $1 \times 10^{-5}$. The Reynolds-averaged N-S equation for Taylor-Couette flow was solved numerically using the realizable $k-\varepsilon$ model combined with the standard wall function. The numerical result of the velocity distribution is in good agreement with the experimental results. Additionally, the dynamic equation for the particle number distribution function was solved numerically using the Taylor series expansion moment method (TEMOM). The variation characteristics of particle number density, diameter and polydispersity in the flow were analyzed. The results show that particle breakage is obvious in the region with strong vorticity due to the large shear strength, which leads to a significant change in the particle number density, diameter and polydispersity. Furthermore, the effects of the gap width between two cylinders and the Reynolds number on the distribution of the particle number density, size and polydispersity are discussed.
\end{abstract}

Keywords: nanoparticle-laden flow; coagulation; breakage; Taylor-Couette flow; particle distribution

\section{Introduction}

Nanoparticle-laden flow exists in industrial applications and natural phenomena. In the process of transport, particles collide with each other under the influence of the convection and diffusion of the flow, resulting in particle coagulation, breakage and deposition. Research on the variation characteristics of the particle number density, diameter and polydispersity, considering particle coagulation and breakage, under turbulent conditions is significant in applications.

A large number of research papers on particle coagulation have been published. For example, the results in turbulent circular tube flows [1] and shear flows [2] showed that nanoparticle coagulation in turbulent flows was mainly related to the collision caused by the Brownian motion of particles. In fact, the mechanism of particle coagulation includes the Brownian motion of particles and the shear effect of turbulent flow, and which of the two factors is dominant dends on the particle size and turbulence intensity [3,4]. The experimental and numerical results presented by $\mathrm{Yu}$ et al. [5] showed that patterns of coagulation for micron and submicron particles were slightly different due to different particle dynamics. In the case of a sufficiently high turbulence intensity, particles with a diameter of $100 \mathrm{~nm}$ will be turbulently coagulated [4].

Relatively speaking, there have been fewer research papers on particle breakage in turbulent flow. Gan et al. [6] showed that particle breakage was caused by the turbulent shear effect. Yuan et al. [7] studied nanoparticles ejected by high-pressure vessels and found that, under the effect of strong turbulent shear at the nozzle, the particle group would be broken into several hundred nanometers, and even into a smaller scale under the action of secondary pressure dispersion. Ammar et al. [8] analyzed the mechanism related to particle breakage in turbulent pipe flow and found that the breakup was slower in the pipe flow where the turbulent shear rate was lower than in the free jet zone. However, the 
particles would break instantly under the action of high local shear stress. Soos et al. [9] studied particle breakage in turbulent flows by population balance modeling and static light scattering. Baldyga et al. [10] applied population balance modeling with kinetics including the effects of breakage on turbulent flow. Far et al. [11] studied the effect of the fractal dimension and the number of primary particles on particle breakage and found that particles with a fractal number of 1.85 had Stokes numbers smaller than one such that the load on these particles was dominated by the strain rate. Lin et al. [12] studied the penetration efficiency of nanoparticles flowing through a pipe bend considering particle breakage. Amani et al. [13] indicated that the addition of nanoparticles had an obvious effect on drop breakage and consequently their size distribution. Conchuir et al. [14] presented new aggregate stability maps which could be used to discriminate regions in the parameter space where aggregates are fully stable upon both restructuring and breakup, and the parameter space includes the fractal dimension, size and shear rate.

The literature survey reveals that there are few studies on the distribution of nanoparticles in turbulent Taylor-Couette flows considering particle coagulation and breakage. The Taylor-Couette flow is a typical flow, and it has some practical applications [15]. The flow is used in research because it can be controlled at given known local conditions. Wang et al. [16] studied the effects of the shear rate on the breakage of flocs or aggregates consisting of $10 \mu \mathrm{m}$ particles in a turbulent Taylor-Couette flow and demonstrated that computational fluid dynamics could be used to simulate the particle breakage over a wide range of Reynolds numbers considering the heterogeneity of the flow. The research of Wang et al. [16] mainly focused on the change in the fractal dimension and particle size with time at Reynolds numbers changing from 34 to 220. In this work, therefore, a numerical simulation was performed to explore the spatial distribution of the particle number density, size and polydispersity at higher Reynolds numbers up to 16,923. Meanwhile, the characteristics of particle breakage and the effects of the gap width between two cylinders and the Reynolds number on the distribution of the particle number density, size and polydispersity in a turbulent Taylor-Couette flow are discussed. This paper focused on the fragmentation of nanoparticles, which has been ignored in previous studies, and it is of great significance to the study of the particle equilibrium mechanism.

\section{Governing Equations}

\subsection{Equations of Turbulent Taylor-Couette Flow}

A turbulent Taylor-Couette flow is shown in Figure 1, where a cylindrical coordinate system is used. The origin of the coordinate system is set at the center of the bottom surface of the cylinder.

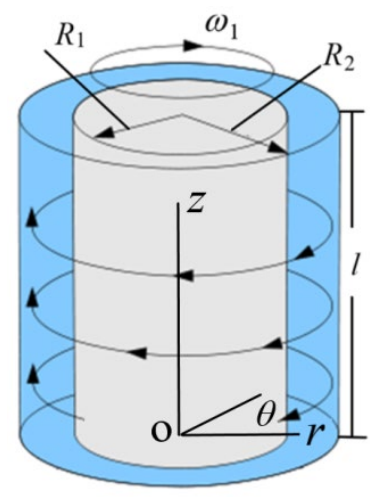

Figure 1. Schematic diagram of turbulent Taylor-Couette flow and coordinate system.

For the incompressible flow, the continuity and the Reynolds-averaged N-S equations are

$$
\frac{\partial u_{i}}{\partial x_{i}}=0
$$




$$
\rho\left(\frac{\partial u_{i}}{\partial t}+u_{k} \frac{\partial u_{i}}{\partial x_{k}}\right)=-\frac{\partial p}{\partial x_{i}}+\frac{\partial}{\partial x_{j}}\left(\mu \frac{\partial u_{i}}{\partial x_{j}}\right)+\frac{\partial}{\partial x_{j}}\left(-\rho \overline{u_{i}^{\prime} u_{j}^{\prime}}\right)
$$

where $u_{i}$ is the mean fluid velocity, $\rho$ is the fluid density, $u^{\prime}$ is the fluctuating velocity, $p$ is the pressure and $\mu$ is the fluid viscosity, and the Reynolds stress $R_{i j}$ is

$$
R_{i j}=-\rho \overline{u_{i}{ }^{\prime} u_{j}^{\prime}}=\rho v_{t}\left(\frac{\partial u_{i}}{\partial x_{j}}+\frac{\partial u_{j}}{\partial x_{i}}\right)-\frac{2}{3} \rho k \delta_{i j}
$$

where $v_{t}=C_{\mu} k^{2} / \varepsilon\left(C_{\mu}\right.$ is a function of the average strain rate, and $k$ and $\varepsilon$ are the turbulent kinetic energy and dissipation rate), and $\delta_{i j}$ is the Kronecker delta function.

The realizable $k-\varepsilon$ turbulence model with swirling correction is selected for a flow with an obvious cyclone. The transport equations of the turbulent kinetic energy and dissipation rate are, respectively,

$$
\begin{gathered}
\frac{\partial}{\partial t}(\rho k)+\frac{\partial}{\partial x_{i}}\left(\rho k u_{i}\right)=\frac{\partial}{\partial x_{i}}\left[\left(\mu+\frac{\mu_{i}}{\sigma_{k}}\right) \frac{\partial k}{\partial x_{j}}\right]+G_{k}-\rho \varepsilon \\
\frac{\partial}{\partial t}(\rho \varepsilon)+\frac{\partial}{\partial x_{j}}\left(\rho \varepsilon u_{j}\right)=\frac{\partial}{\partial x_{j}}\left[\left(\mu+\frac{\mu_{i}}{\sigma_{s}}\right) \frac{\partial \varepsilon}{\partial x_{j}}\right]+\rho C_{1} S \varepsilon-\rho C_{2} \frac{\varepsilon^{2}}{k+\sqrt{v \varepsilon}} \\
C_{1}=\max \left[0.43, \frac{\eta}{\eta+5}\right], \eta=S \frac{k}{\varepsilon}, \mathrm{S} \equiv \sqrt{2 \Omega_{i j} \Omega_{i j}}, \Omega_{i j}=\frac{1}{2}\left(\frac{\partial u_{j}}{\partial x_{i}}-\frac{\partial u_{i}}{\partial x_{j}}\right), G_{k}=-\rho \overline{u_{i}^{\prime} u_{j}^{\prime}} \frac{\partial u_{j}}{\partial x_{i}}
\end{gathered}
$$

where $G_{k}$ represents the turbulent kinetic energy generated by the average velocity gradient, $C_{2}=1.9, C_{1 \varepsilon}=1.44, \sigma_{k}=1.0$ and $\sigma_{\varepsilon}=1.2$.

\subsection{Population Balance Equation (PBE) for Nanoparticles}

Nanoparticles follow the flow field, collide with others, coagulate under Brownian motion and shear force and break up at a large shear force. The nanoparticles are treated as a continuum, and the instantaneous PBE [17] for nanoparticles under the combined effects of fluid convection, particle diffusion, coagulation and breakage is

$$
\begin{array}{r}
\frac{\partial n(v, t)}{\partial t}+\nabla \cdot n(v, t) u-\nabla \cdot D \nabla n(v, t)=\frac{1}{2} \int_{0}^{v} \beta\left(v_{1}, v-v_{1}\right) n\left(v_{1}, t\right) n\left(v-v_{1}, t\right) \mathrm{d} v_{1} \\
-\int_{0}^{\infty} \beta\left(v_{1}, v\right) n(v, t) n\left(v_{1}, t\right) \mathrm{d} v_{1}+\int_{v}^{\infty} a\left(v_{1}\right) b\left(v \mid v_{1}\right) n\left(v_{1}\right) \mathrm{d} v_{1}-a(v) n(v)
\end{array}
$$

where $n(v, t)$ is the particle volume distribution function at time $t, \boldsymbol{u}$ is the fluid velocity vector, $D$ is the turbulent diffusion coefficient, which is usually approximated by the turbulent viscosity of the fluid [18], $\beta\left(v_{1}, v\right)$ is the volume-based coagulation kernel for two particles with volumes $v$ and $v_{1}, a(v)$ is the volume-based breakage kernel that provides the frequency of breakage of a particle of volume $v$ and $b\left(v \mid v_{1}\right)$ is the breakage distribution function.

Assuming that particle coagulation is the result of Brownian motion and turbulent shear, then $\beta$ is

$$
\beta=\beta_{B}+\beta_{T}
$$

The coagulation kernel caused by Brownian motion is [19]

$$
\beta_{B}=\frac{2 k_{B} T}{3 \mu}\left(\frac{1}{v^{1 / 3}}+\frac{1}{v_{1}^{1 / 3}}\right)\left(v^{1 / 3}+v_{1}^{1 / 3}\right)+\frac{2 k_{B} T}{3 \mu} \frac{1.591 \lambda}{(3 / 4 \pi)^{1 / 3}}\left(\frac{1}{v^{2 / 3}}+\frac{1}{v_{1}^{2 / 3}}\right)\left(v^{1 / 3}+v_{1}^{1 / 3}\right)
$$

where $k_{B}$ is the Boltzmann constant, $T$ is the temperature, $\mu$ is the fluid viscosity and $\lambda$ is the gas molecular mean free path. 
Based on isotropic turbulence theory, Saffman [20] deduced the particle collision rate under turbulent shear; correspondingly, the coagulation kernel caused by turbulent shear is

$$
\beta_{T}=\sqrt{\frac{3}{10 \pi}}\left(v^{1 / 3}+v_{1}^{1 / 3}\right)^{3}\left(\frac{\varepsilon}{v}\right)^{1 / 2}
$$

The volume-based breakage kernel is [21]

$$
a(v)=0.0047\left(\frac{\varepsilon}{v}\right)^{0.8} v^{1 / 3}
$$

where $\varepsilon$ is the turbulent dissipation rate, and $v$ is the fluid viscosity.

\subsection{Taylor Series Expansion Moment Method (TEMOM)}

Using the moment method to solve PBEs numerically has the characteristics of a high computational efficiency and easy implementation while ensuring accuracy [22]. Moreover, the particle number, volume, mass and polydispersity can be obtained simultaneously by using the moment method. Equation (6) can be transformed into a moment equation by multiplying the terms of Equation (6) by $v_{k}$ and then integrating them over the entire volume distribution:

$$
\begin{aligned}
\frac{\partial m_{k}}{\partial t}+\nabla \cdot m_{k} \boldsymbol{u}-\nabla \cdot D \nabla m_{k}=\frac{1}{2} \int_{0}^{\infty} \int_{0}^{\infty}[ & {\left[\left(v+v_{1}\right)^{k}-v^{k}-v_{1}^{k}\right] \beta\left(v_{1}, v\right) n(v, t) n\left(v_{1}, t\right) \mathrm{d} v \mathrm{~d} v_{1} } \\
& +\int_{0}^{\infty} v^{k} \int_{v}^{\infty} a\left(v_{1}\right) b\left(v \mid v_{1}\right) n\left(v_{1}\right) \mathrm{d} v_{1} \mathrm{~d} v-\int_{0}^{\infty} v^{k} a(v) n(v) \mathrm{d} v
\end{aligned}
$$

The $k$ th moment of the particle distribution function is defined by

$$
m_{k}=\int_{0}^{\infty} v^{k} n(v, t) d v
$$

where $M_{0}$ is the total particle number, $M_{1}$ represents the particle volume and is proportional to the particle mass and $M_{2}$ is directly relevant to the particle polydispersity.

In this paper, the TEMOM [22,23] was used to close Equation (11). The basic idea of the TEMOM is to transform any moment into the form of the first three moments using the Taylor series expansion technique so that the moment equations can be solved in a closed way. The corresponding moment equations are

$$
\begin{aligned}
& \frac{\partial m_{0}}{\partial t}+\nabla \cdot m_{0} \boldsymbol{u}-\nabla \cdot D \nabla m_{0} \\
& =-\sqrt{\frac{3}{10 \pi} \frac{\varepsilon}{v}} \frac{m_{0}^{3} m_{2}^{2}-20 m_{0}^{3} m_{1}^{2} m_{2}+127 m_{0} m_{1}^{4}}{27 m_{1}^{3}}-0.0047\left(\frac{\varepsilon}{v}\right)^{0.8} \frac{m_{0}^{5 / 3} m_{2}-10 m_{0}^{2 / 3} m_{1}^{2}}{9 m_{1}^{5 / 3}} \\
& +\frac{2 k T}{3 \mu}\left[\frac{2 m_{0}^{4} m_{2}^{2}-13 m_{0}^{3} m_{1}^{2} m_{2}-151 m_{0}^{2} m_{1}^{4}}{81 m_{1}^{4}}-\frac{1.591 \lambda}{(3 / 4 \pi)^{1 / 3}} \frac{-5 m_{0}^{13 / 3} m_{2}^{2}+64 m_{0}^{10 / 3} m_{1}^{2} m_{2}+103 m_{0}^{10 / 3} m_{1}^{4}}{81 m_{1}^{1 / 3}}\right] \\
& \frac{\partial m_{1}}{\partial t}+\nabla \cdot m_{1} \boldsymbol{u}-\nabla \cdot D \nabla m_{1}=0 \\
& \frac{\partial m_{2}}{\partial t}+\nabla \cdot m_{2} \boldsymbol{u}-\nabla \cdot D \nabla m_{2} \\
& =-\sqrt{\frac{3}{10 \pi} \frac{\varepsilon}{v}} \frac{4\left(5 m_{0}^{2} m_{2}^{2}+14 m_{1}^{4}+35 m_{0} m_{1}^{2} m_{2}\right)}{27 m_{0} m_{1}}-0.0047\left(\frac{\varepsilon}{v}\right)^{0.8} \frac{14 m_{0} m_{1}^{1 / 3} m_{2}-5 m_{1}^{7 / 3}}{18 m_{0}^{4 / 3}} \\
& +\frac{4 k T}{3 \mu}\left[\frac{2 m_{0}^{4} m_{2}^{2}-13 m_{0} m_{1}^{2} m_{2}-151 m_{1}^{4}}{81 m_{1}^{2}}+\frac{1.591 \lambda}{(3 / 4 \pi)^{1 / 3}} \frac{-4 m_{0}^{7 / 3} m_{2}^{2}+8 m_{0}^{4 / 3} m_{1}^{2} m_{2}+320 m_{0}^{1 / 3} m_{1}^{4}}{81 m_{1}^{7 / 3}}\right]
\end{aligned}
$$




\section{Numerical Simulation}

\subsection{Numerical Model}

In the numerical simulation, initially, the particles were spherical particles with a diameter of $200 \mathrm{~nm}$ and a volume fraction of $1 \times 10^{-5}$. The one-way coupling method was adopted, i.e., the influence of particles on the flow is ignored. The Reynolds number was defined as $R e=\omega_{1} \times R_{1} \times\left(R_{2}-R_{1}\right) / \nu$. Table 1 summarizes the parameters used in the computation under different conditions. The governing Equations (13)-(15) of particles and the corresponding Equations (1)-(5) of turbulent flow were solved using the finite volume method in OpenFOAM-5, and the term of velocity-pressure coupling and the convection term were dealt with using the Open FOAM SIMPLE algorithm. The equations for particles were solved numerically with a self-made program. The no-slip boundary condition was applied to the top and bottom walls as well as the wall of the outer cylinder.

Table 1. Parameters used in the computation under different conditions.

\begin{tabular}{|c|c|c|c|c|c|}
\hline Case & Radius of Outer Cylinder $R_{2}(\mathrm{~m})$ & $R=R_{1} / R_{2}$ & Angular Velocity $\omega_{1}(\mathrm{rad} / \mathrm{s})$ & Reynolds Number & Particle Breakage \\
\hline 0 & 4.760 & 0.733 & 31.718 & 13,520 & no \\
\hline 1 & 4.760 & 0.733 & 31.718 & 13,520 & yes \\
\hline 2 & 5.584 & 0.625 & 31.718 & 13,520 & yes \\
\hline 3 & 4.363 & 0.800 & 31.718 & 13,520 & yes \\
\hline 4 & 4.760 & 0.733 & 10.000 & 4263 & yes \\
\hline 5 & 4.760 & 0.733 & 19.830 & 8453 & yes \\
\hline 6 & 4.760 & 0.733 & 39.700 & 16,923 & yes \\
\hline
\end{tabular}

\subsection{Mesh Independence Test and Validation}

The grid system consisted of $20(r) \times 70(\theta) \times 500(z)=700,000$ grid points. A uniform grid was used in three directions. A grid independence test was performed by changing grid points to 1.2 times in all directions. The results show that the same 16 pairs of vortices can be observed on any axial plane before and after mesh refinement, as shown in Figure 2.

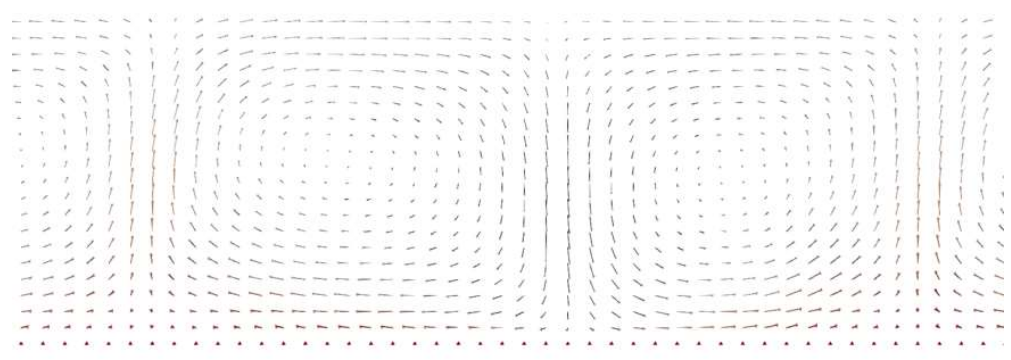

Figure 2. Velocity vector fields with pairs of vortices.

In order to compare the numerical results with the experimental results, as shown in Figure 1, the outer cylinder with a radius of $4.76 \mathrm{~cm}$ was fixed, and the inner cylinder with a radius of $3.49 \mathrm{~cm}$ rotated at $31.718 \mathrm{rad} / \mathrm{s}$. The fluid viscosity $\mu$ was $1.097 \times 10^{-3} \mathrm{~kg} / \mathrm{m} \cdot \mathrm{s}$, the particle density was the same as the fluid density, namely, $1055 \mathrm{~kg} / \mathrm{m}^{3}$, and the Reynolds number was 13,520.

To validate the numerical method used in the simulation, we compared the present numerical results of the axial and radial velocities at the position of $0.0394 \mathrm{~m}$ on the central axial plane with the experimental results [16], as shown in Figure 3. It can be seen that both results are basically consistent.

A grid independence test was performed by changing the grid points. Figure 4 shows the comparison of the velocity profiles between the numerical results with a coarse mesh (230,720 cells) and fine mesh (518,400 cells) and the experimental results [24] in a chamber. Almost no difference is observed for the cases of the coarse and fine meshes. In addition, the agreement between the numerical and experimental results indicates that the numerical method is reasonable and reliable. 


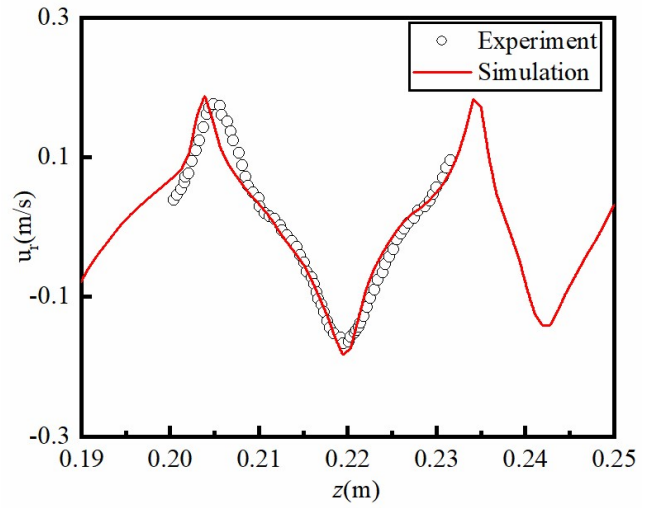

(a)

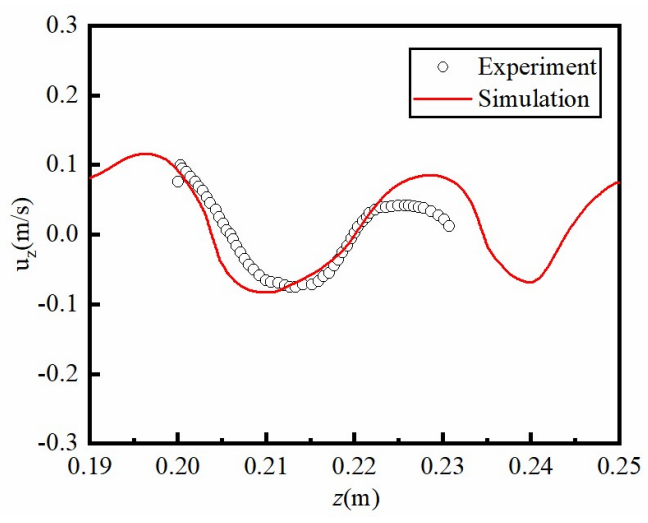

(b)

Figure 3. Comparison of experimental and predicted mean velocities. ( $\bigcirc$ : experiment data; - : simulation). (a) radial velocity, (b) axial velocity.

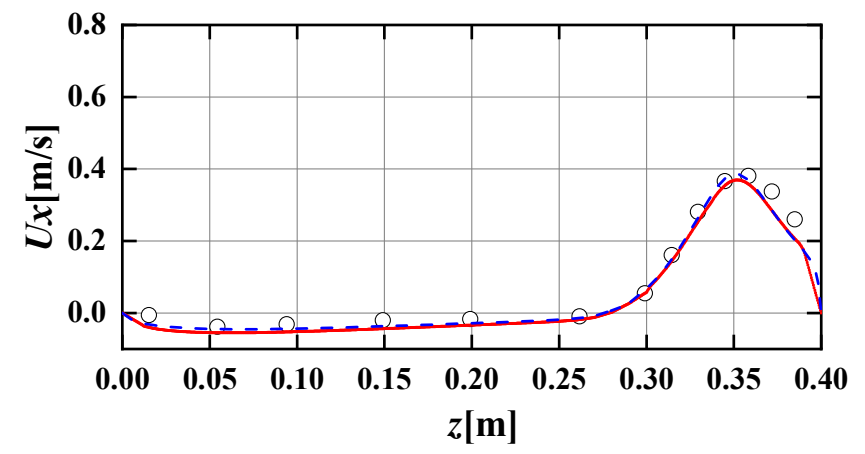

Figure 4. Comparison of velocity profiles between numerical and experimental results. $\bigcirc$ : experiment data; solid line: simulation with coarse mesh; dashed line: simulation with fine mesh.

\section{Results and Discussion}

\subsection{Difference between Considering and Not Considering Particle Breakage}

In Equation (11), if the last two terms on the right-hand side of the equation are retained or ignored, the difference between considering and not considering the particle breakage can be shown.

\subsubsection{Particle Number Density}

Define a dimensionless particle number density $M_{0}=m_{0} / m_{00}$, where $m_{0}$ and $m_{00}$ are the particle number density and initial particle number density, respectively. As it can be seen from the contour of $M_{0}$ on the $r-z$ plane in Figure 5, there is no obvious difference in the distributions of the particle number density when considering and not considering the particle breakage, i.e., the particle breakage is weak when taking the parameters of case 1 in Table 1. As shown in Figure 5, influenced by the vortex structure, particles are concentrated in the area with a large velocity in the form of vortex pairs, and the particle distribution is not uniform or symmetrical. The regions with the smallest particle number density appear on the two ends of the cylinder, where the shear rate is much smaller. This is attributed to the influence of particle inertia force and particle coagulation. The change in the particle number density is related to the particle coagulation and breakage. Figure 6 shows the change in $M_{0}$ with time. We can see that the particles coagulate violently in the initial stage, resulting in a sharp decrease in the particle number density. The particle number density reaches a stable value with the passage of time because the particle coagulation and breakage reach an equilibrium. 


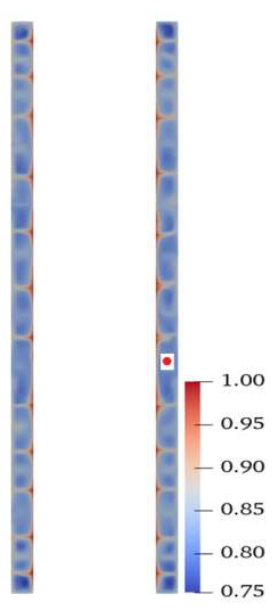

(a)
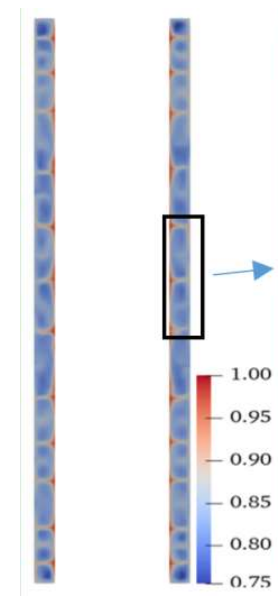

Figure 5. Contour of $M_{0}$ with and without considering breakage. (a) without breakage (case 0 in Table 1), (b) breakage (case 1 in Table 1).

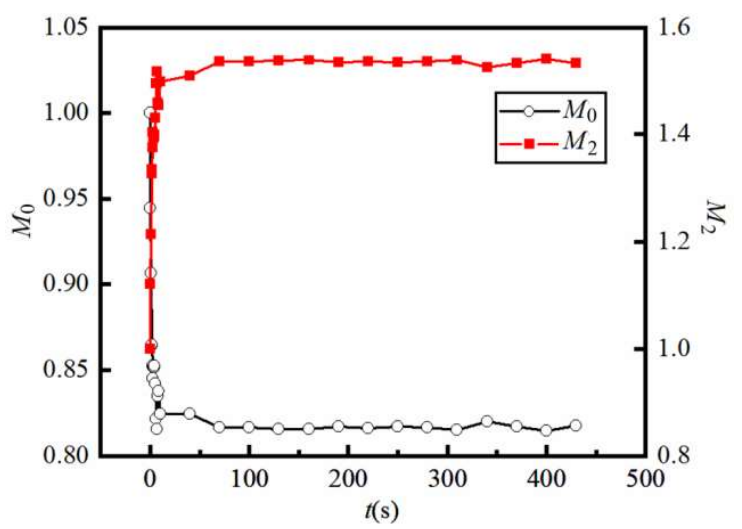

Figure 6. Evolution of $M_{0}$ and $M_{2}$ with time at red point in Figure 4 (case 1 in Table 1 ).

$M_{2}$ is directly relevant to the particle polydispersity. As shown in Figure 6, the particle polydispersity increases sharply at first because of the higher particle coagulation rate and then reaches a stable value.

\subsubsection{Particle Diameter}

Single size particles are initially distributed in the flow field. Due to the influence of particle coagulation and breakage, the average size of particles will change. Define the geometric mean diameter of particles as [25]

$$
d_{g}=\left(\frac{m_{1}^{2}}{\sqrt{m_{0}^{3} m_{2}}}\right)^{1 / 3}
$$

Distributions of the particle diameter along the axial direction are shown in Figure 7, where both cases considering and not considering the particle breakage are presented. We can see that the distributions of the particle diameter change along the axial direction and are closely related to the velocity distribution of the flow field. The peaks of the particle diameter and velocity basically coincide, as shown in Figure $4 \mathrm{~b}$. In the area close to the cylinder wall, the energy dissipation of the flow is larger, and the particle diameter is smaller. The peak value of the particle diameter distribution is closer to the outer wall. The 
distribution of the particle diameter considering the particle breakage is different from that without considering the particle breakage, and the values of the former are smaller.

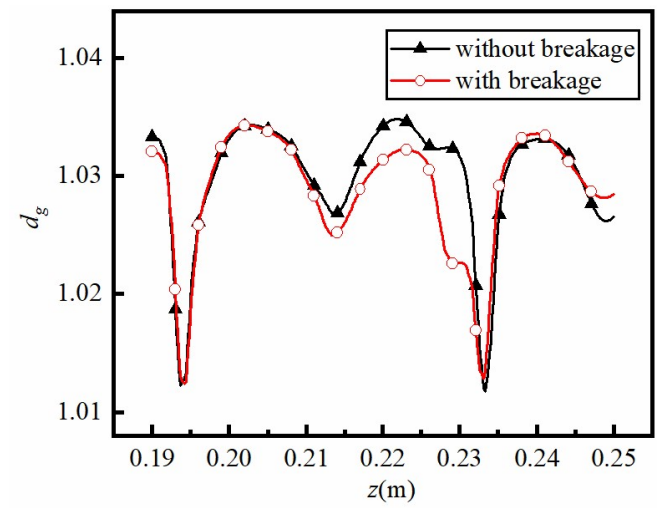

(a)

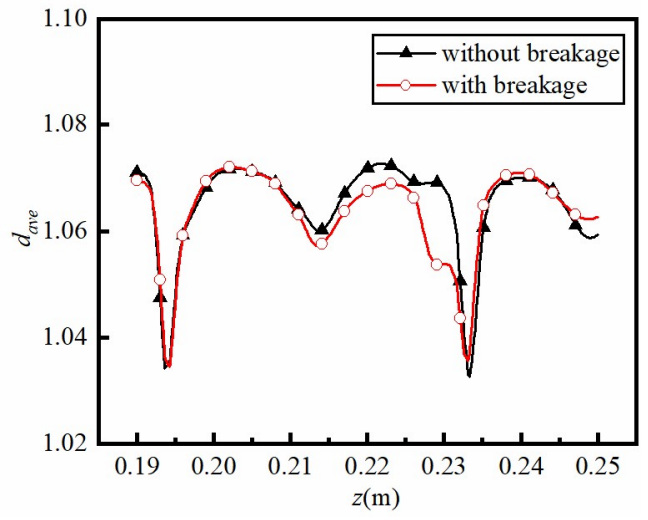

(b)

Figure 7. Distribution of particle diameter along the axial direction (cases 0 and 1 in Table 1). (a) geometric mean diameter, (b) mean diameter.

\subsubsection{Particle Polydispersity}

The coagulation and breakage of particles cause the initial single particle scale to become multi-scale, resulting in an increase in the particle polydispersity. Define the particle polydispersity with the following standard deviation $\sigma$ of the particle distribution:

$$
\ln ^{2} \sigma=\frac{1}{9} \ln \left(\frac{m_{0} m_{2}}{m_{1}^{2}}\right)
$$

Thus, the greater the $\sigma$, the stronger the particle polydispersity.

Figure 8 shows the distribution of the standard deviation of the particle distribution along the axial direction. It can be seen that the values of $\sigma$ are smaller for the case considering the particle breakage than that without considering the particle breakage. The reason is that if the particles only coagulate without breaking, the particle diameter after coagulation will increase monotonically, resulting in a monotonic increase in the particle polydispersity. However, if the particles are both coagulated and broken, the diameter range of particles becomes smaller compared with the case without considering the particle breakage, meaning that the particle polydispersity is reduced.

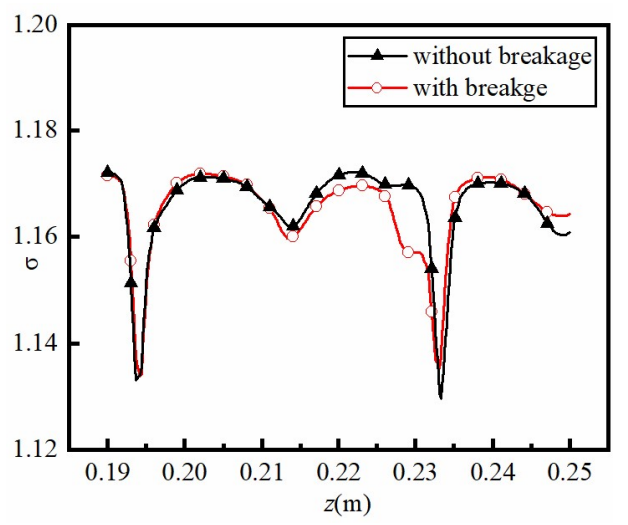

Figure 8. Distribution of standard deviation of particle distribution (cases 0 and 1 in Table 1). 


\subsection{Effect of the Gap Width between Two Cylinders}

The gap width between two cylinders will affect the flow characteristics, thus affecting the distribution of particles.

\subsubsection{Particle Number Density}

The distribution of $M_{0}$ is affected by the vortex structure and presents quasi-periodicity along the axial direction. The larger the gap width, the fewer vortex pairs there are. The distribution of $M_{0}$ changes along the radial direction, and the largest values of $M_{0}$ appear near the wall of the inner cylinder and present periodicity along the axial direction. The difference in the gap width leads to the difference in the vortex structure and even the difference in the distribution of $M_{0}$. The values of $M_{0}$ increase with an increasing gap width because particle coagulation is less likely to occur at a wider gap. The difference in the values of $M_{0}$ becomes smaller along the axial direction with the increase in the gap width due to the large axial velocity.

\subsubsection{Particle Size and Polydispersity}

Figure 9 shows the distributions of the particle diameter along the axial direction for different gap widths. It can be seen that the distributions of the particle diameter show a quasi-periodic variation along the axial direction. The particle diameter decreases with the decreasing gap width. The reason is that the walls of the two cylinders are closer when the gap width is smaller, and there is a stronger shear in the flow field, which makes the particles easier to break up.

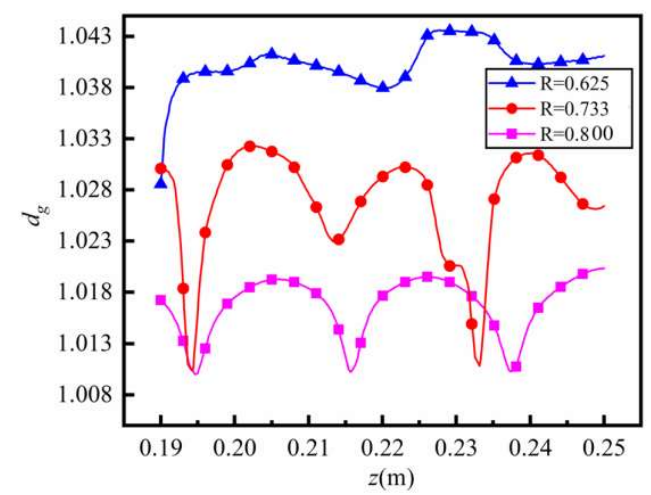

(a)

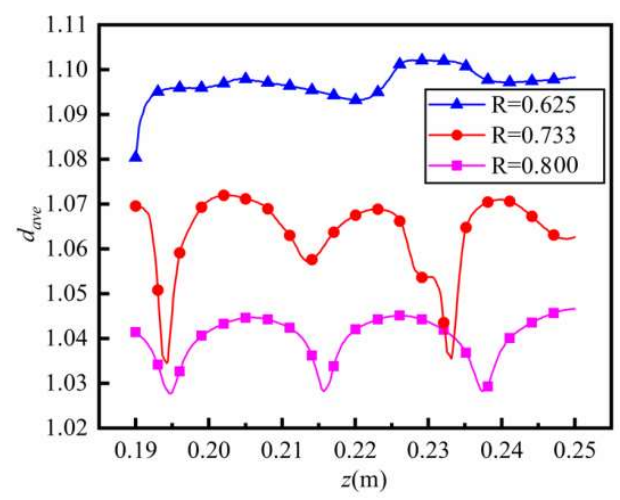

(b)

Figure 9. Distribution of particle diameter for different gap widths (cases 1-3 in Table 1). (a) geometric mean diameter, (b) mean diameter.

Distributions of the standard deviation of the particle distribution along the axial direction are shown in Figure 10, where the values of $\sigma$ show the same change trend as the particle diameter shown in Figure 9. The standard deviation of the particle distribution, i.e., the particle polydispersity, increases with the increase in the gap width, which is attributed to the idea that, on the one hand, particle breakage is less likely to occur in the flow with a weaker shear when the gap is wider, and, on the other hand, the particles size increases continuously due to coagulation, and finally the size range of the particles becomes wider. 


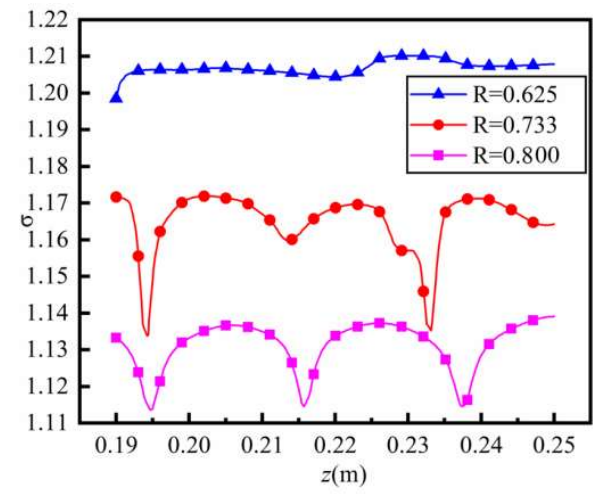

Figure 10. Distribution of standard deviation of particle distribution (cases 1-3 in Table 1).

\subsection{Effect of Reynolds Number}

The Reynolds number is related to the angular velocity of the inner cylinder, gap width between two cylinders and viscosity coefficient of the fluid. Different Reynolds numbers will lead to different particle distributions.

\subsubsection{Particle Number Density}

Axial distributions of the particle number density $M_{0}$ in the middle position of the inner and outer cylinders are shown in Figure 11. We can see that the values of $M_{0}$ basically vary between 0.75 and 0.9 when $R e=13,520$ and 16,923 . This means that the change in the Reynolds number has little effect on the distribution of the particle number density when the $R e$ is large. The reason may be attributed to the idea that the characteristics of the flow field and vortex structure tend to be stable when the Reynolds number exceeds a certain value, and the distribution of the particle number density is affected by the characteristics of the flow. In Figure 11, the values of $M_{0}$ increase when the Reynolds number changes from 4264 to 13,520. A large Reynolds number means that the turbulence effect is stronger. As it can be seen from expressions (9) and (10), the coagulation and kernel breakage caused by turbulent shear are directly proportional to the turbulent dissipation rate $\varepsilon$. The stronger turbulence effect will promote particle coagulation and breakage. Particle coagulation reduces and particle breakage increases the particle number density. However, the coagulation kernel is proportional to the 0.5 th power of $\varepsilon$, while the breakage kernel is proportional to the 0.8 th power of $\varepsilon$, as shown in expressions (9) and (10). In general, the effect of turbulence on particle breakage is greater than that on particle coagulation, meaning that the values of $M_{0}$ increase with an increasing Reynolds number.

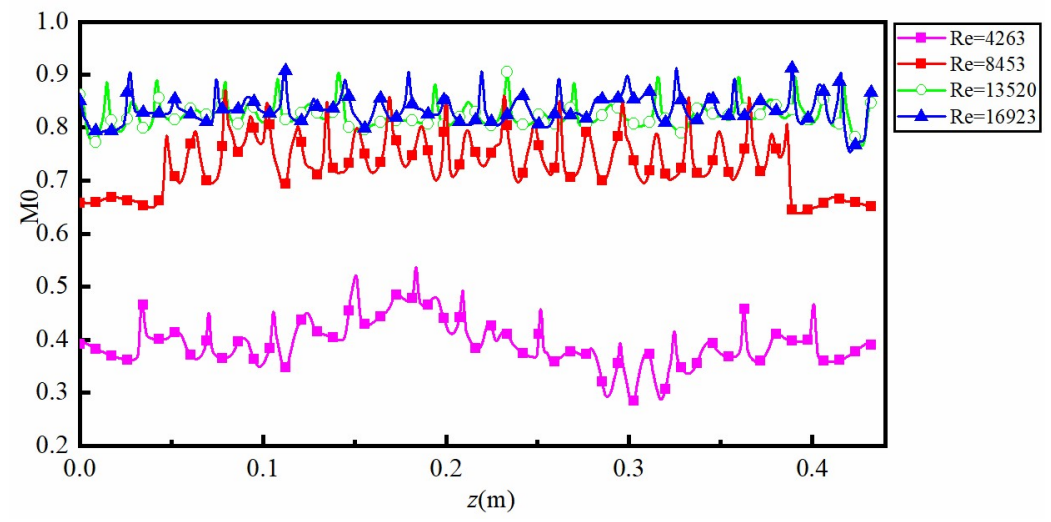

Figure 11. Axial distribution of $M_{0}$ in the middle position of the inner and outer cylinders. $R e=4363$ (case 4 in Table 1), $R e=8453$ (case 5), $\operatorname{Re}=13,520$ (case 1), $R e=16,923$ (case 6). 


\subsubsection{Particle Size and Polydispersity}

Figures 12 and 13 show the distributions of the geometric mean diameter of particles and the standard deviation of the particle distribution along the axial direction, respectively. It can be seen that the values of $d_{g}$ and $\sigma$ for $R e=4263$ are far greater than the values of $d_{g}$ and $\sigma$ for other $R e$, which is consistent with the conclusion in Figure 11. The geometric mean diameter of particles is generally larger when the Re is low because the slower the cylinder rotates, the longer the time for particles to collide and coagulate with others. Moreover, a low Re means that particles are less likely to break up due to the low shear rate. In Figure 13, the standard deviation of the particle distribution, i.e., the particle polydispersity, increases with the decrease in Re. On the one hand, particle breakage is less likely to occur in the flow with a weaker shear when the Re is lower. On the other hand, the particles have more time to collide and coagulate in the flow with a lower $R e$, resulting in a continuous increase in the particle diameter, and finally the size range of the particles becomes wider.

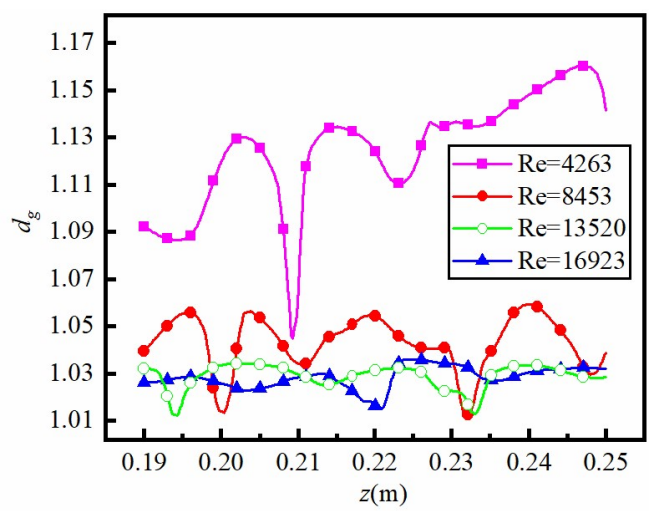

Figure 12. Distribution of geometric mean diameter. $R e=4363$ (case 4 in Table 1), $R e=8453$ (case 5), $\operatorname{Re}=13,520$ (case 1), $R e=16,923$ (case 6).

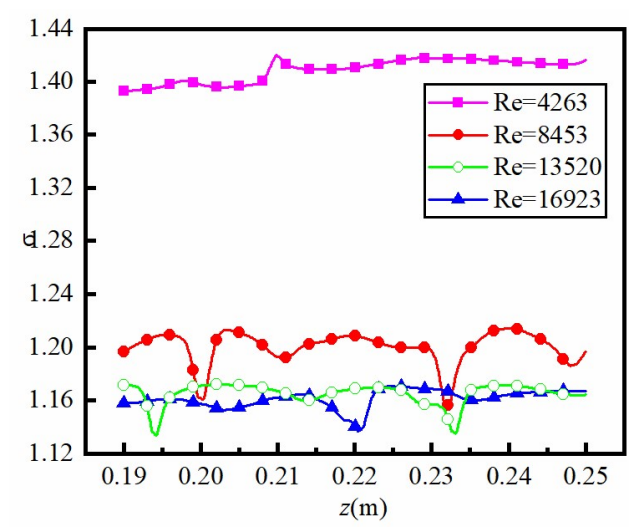

Figure 13. Distribution of standard deviation. $R e=4363$ (case 4 in Table 1), $R e=8453$ (case 5), $\operatorname{Re}=13,520$ (case 1), $R e=16,923$ (case 6).

\section{Conclusions}

In this paper, the Taylor series expansion moment method was used to numerically simulate the dynamic evolution of nanoparticles in a turbulent Taylor-Couette flow. The characteristics of particle breakage and the influences of the gap width between two cylinders and the Reynolds number on the distribution of the particle number density, diameter and polydispersity were analyzed. The main conclusions are summarized as follows:

(1) Particle breakage leads to an increase in the particle number density and a decrease in the particle mean diameter, but it has no obvious effect on the particle polydispersity. 
(2) The coagulation and breakage of particles reach a balance when the system lasts for a period of time. Accordingly, the particle number density, diameter and polydispersity tend to a steady state.

(3) The gap width between two cylinders has an effect on the flow field, vortex structure and even particle distribution. The values of the particle number density, particle diameter and polydispersity increase with an increasing gap width. The difference in the values of the particle number density becomes smaller along the axial direction with an increase in the gap width.

(4) The change in the Reynolds number has little effect on the distribution of the particle number density when the $R e$ is large. The effect of turbulence on particle breakage is greater than that on particle coagulation. With an increase in the Reynolds number, the particle number density increases, but the particle diameter and polydispersity decrease.

Author Contributions: Conceptualization, R.S.; methodology, H.Y.; software, H.Y. and R.S.; validation, R.S.; formal analysis, R.S.; investigation, R.S.; resources, R.S. and H.Y. and J.L.; data curation, R.S.; writing — original draft preparation, R.S.; writing—review and editing, J.L.; visualization, R.S.; supervision, J.L.; project administration, J.L.; funding acquisition, J.L. All authors have read and agreed to the published version of the manuscript.

Funding: National Natural Science Foundation of China (Grant 91852102).

Institutional Review Board Statement: Not applicable.

Informed Consent Statement: Not applicable.

Data Availability Statement: Data sharing not applicable.

Conflicts of Interest: The authors declare no conflict of interest.

\section{References}

1. Zhu, J.; Qi, H.; Wang, J. Nanoparticle dispersion and coagulation in a turbulent round jet. Int. J. Multiph. Flow 2013, 54, 22-30.

2. Garrick, C.S. Effects of Turbulent fluctuations on nanoparticle coagulation in shear flows. Aerosol Sci. Technol. 2011, 45, 1272-1285. [CrossRef]

3. He, Q.I.; Xie, M.L. Thermodynamic analysis of Brownian motion-induced particle agglomeration using the taylor-series expansion. Processes 2021, 9, 1218. [CrossRef]

4. Park, S.H.; Kruis, F.E.; Lee, K.W.; Fissan, H. Evolution of particle size distributions due to turbulent and brownian coagulation Aerosol Sci. Technol. 2002, 36, 419-432. [CrossRef]

5. Yu, Z.; Bu, S.; Zhang, L.; Wu, R.; Chen, F.Y.; Xu, W.G.; Liu, L.; Zhao, Y.H.; Xu, Y.S. Turbulent coagulation of micron and submicron particles in swirling flow. Separ. Purif. Technol. 2020, 248, 117098. [CrossRef]

6. Gan, F.; Lin, J.Z.; Yu, M. Particle size distribution in a planar jet flow undergoing shear-induced coagulation and breakage. J. Hydrodynam. 2010, 22, 445-455. [CrossRef]

7. Yuan, F.; Tu, C.; Yu, J.; Cui, Z.W. High-pressure dispersion of nanoparticle agglomerates through a continuous aerosol disperse. Appl. Nanosci. 2019, 9, 1857-1868. [CrossRef]

8. Ammar, Y.; Dehbi, A.; Reeks, M.W. Break-up of aerosol agglomerates in highly turbulent gas flow. Flow Turbul. Combust. 2012, 89, 465-489. [CrossRef]

9. Soos, M.; Sefcik, J.; Morbidelli, M. Investigation of aggregation, breakage and restructuring kinetics of colloidal dispersions in turbulent flows by population balance modeling and static light scattering. Chem. Eng. Sci. 2006, 61, 2349-2363. [CrossRef]

10. Baldyga, J.; Orciuch, W.; Makowski, L.; Krasinski, A.; Malski-Brodzicki, M.; Malik, K. Shear flow of aggregated nanosuspensionsFundamentals and model formulation. J. Dispers. Sci. Technol. 2008, 29, 564-572. [CrossRef]

11. Far, E.K.; Geier, M.; Kutscher, K.; Krafczyk, M. Simulation of micro aggregate breakage in turbulent flows by the cumulant lattice Boltzmann method. Comput. Fluids 2016, 140, 222-231. [CrossRef]

12. Lin, J.Z.; Yin, Z.Q.; Lin, P.F.; Yu, M.Z.; Ku, X.K. Distribution and penetration efficiency of nanoparticles between 8-550 nm in pipe bends under laminar and turbulent flow conditions. Int. J. Heat Mass Transf. 2015, 85, 61-70. [CrossRef]

13. Amani, P.; Amani, M.; Saidur, R.; Yan, W.M. Hydrodynamic performance of a pulsed extraction column containing ZnO nanoparticles: Drop size and size distribution. Chem. Eng. Res. Design 2017, 121, 275-286. [CrossRef]

14. Conchuir, B.O.; Harshe, Y.M.; Lattuada, M.; Zaccone, A. Analytical model of fractal aggregate stability and restructuring in shear flows. Ind. Eng. Chem. Res. 2014, 53, 9109-9119. [CrossRef]

15. Schrimpf, M.; Esteban, J.; Warmeling, H.; Farber, T.; Behr, A.; Vorholt, A.J. Taylor-Couette reactor: Principles, design, and applications. AIChE J. 2021, 67, e17228. [CrossRef] 
16. Wang, L.; Vigil, R.D.; Fox, R.O. CFD simulation of shear-induced aggregation and breakage in turbulent Taylor-Couette flow. J. Coll. Interf. Sci. 2005, 285, 167-178. [CrossRef]

17. Marchisio, D.L.; Soos, M.; Sefcik, J.; Morbidelli, M. Role of turbulent shear rate distribution in aggregation and breakage processes. AIChE J. 2006, 52, 158-173. [CrossRef]

18. Guichard, R.; Belut, E. Simulation of airborne nanoparticles transport, deposition and aggregation: Experimental validation of a CFD-QMOM approach. J. Aerosol Sci. 2017, 104, 16-31. [CrossRef]

19. Yu, M.; Lin, J.Z. Taylor-expansion moment method for agglomerate coagulation due to Brownian motion in the entire size regime. J. Aerosol Sci. 2009, 40, 549-562. [CrossRef]

20. Saffman, P.G.; Turner, J.S. On the collision of drops in turbulent clouds. J. Fluid Mechan. 1956, 1, 16-30. [CrossRef]

21. Spicer, P.T.; Pratsinis, S.E. Coagulation and fragmentation: Universal steady-state particle-size distribution. AICHE J. 1996, 42, 1612-1620. [CrossRef]

22. Yu, M.; Lin, J.Z.; Chan, T.L. A new moment method for solving the coagulation equation for particles in Brownian motion. Aerosol Sci. Technol. 2008, 42, 705-713. [CrossRef]

23. Yu, M.; Lin, J.Z. Taylor series expansion scheme applied for solving population balance equation. Rev. Chem. Eng. 2018, 34, 561-594. [CrossRef]

24. Belut, E.; Théo, C. A new experimental dataset to validate CFD models of airborne nanoparticles agglomeration. In Proceedings of the 9th International Conference on Multiphase Flow, Firenze, Italy, 22-27 May 2016.

25. Pratsinis, S.E. Simultaneous nucleation, condensation, and coagulation in aerosol reactors. J. Coll. Interf. Sci. 1988, $124,416-427$. [CrossRef] 